\title{
Review
}

\section{Recent advances in modeling and analysis of bioelectric and biomagnetic sources ${ }^{a}$}

\author{
Tilmann H. Sander ${ }^{1, *}$, Thomas R. Knösche ${ }^{2}$, Alois \\ Schlögl ${ }^{3}$, Florian Kohl ${ }^{4}$, Carsten H. Wolters ${ }^{5}$, Jens \\ Haueisen $^{6,7}$ and Lutz Trahms ${ }^{1}$ \\ ${ }^{1}$ Physikalisch-Technische Bundesanstalt, Berlin, Germany \\ ${ }^{2}$ Max Planck Institute for Human Cognitive and Brain \\ Sciences, Leipzig, Germany \\ ${ }^{3}$ Institute for Human Computer Interfaces, Graz University \\ of Technology, Graz, Austria \\ ${ }^{4}$ Electronics and Medical Signal Processing Group, Berlin \\ University of Technology, Berlin, Germany \\ ${ }^{5}$ Institute for Biomagnetism and Biosignal Analysis, \\ University of Münster, Münster, Germany \\ ${ }^{6}$ Biomagnetic Center, Department of Neurology, University \\ of Jena, Jena, Germany \\ ${ }^{7}$ Institute of Biomedical Engineering and Informatics, \\ Ilmenau University of Technology, Ilmenau, Germany
}

\begin{abstract}
Determining the centers of electrical activity in the human body and the connectivity between different centers of activity in the brain is an active area of research. To understand brain function and the nature of cardiovascular diseases requires sophisticated methods applicable to non-invasively measured bioelectric and biomagnetic data. As it is difficult to solve for all unknown parameters at once, several strains of data analysis have been developed, each trying to solve a different part of the problem and each requiring a different set of assumptions. Current trends and results from major topics of electro- and magnetoencephalographic data analysis are presented here together with the aim of stimulating research into the unification of the different approaches. The following topics are discussed: source reconstruction using detailed finite element modeling to locate sources deep in the brain; connectivity analysis for the quantification of strength and direction of information flow between activity centers, preferably incorporating an inverse solution; the

\footnotetext{
aThis contribution is part of the workshop "Biosignal Processing 2008: Innovations in Detection and Analysis of Bioelectric and Biomagnetic Signals" (Potsdam, Germany, July 16-18, 2008). The workshop was organized by the German Society for Biomedical Engineering in VDE (DGBMT). All authors contributed equally to this article.

*Corresponding author: Dr. Tilmann H. Sander, Physikalisch-

Technische Bundesanstalt, Abbestr. 2-12, 10587 Berlin,

Germany

Phone: +49-30-34817436

Fax: +49-30-34817361

E-mail: tilmann.sander-thoemmes@ptb.de
}

conflict between the statistical independence assumption of sources and a possible connectivity; the verification and validation of results derived from non-invasively measured data through animal studies and phantom measurements. This list already indicates the benefits of a unified view.

Keywords: autoregressive modeling; electro-/magnetoencephalography; finite element method; inverse problem; statistical independence; validation.

\section{Introduction}

This paper summarizes a special session of the Biosignal Processing workshop held at University of Potsdam in July 2008. The subject addressed was recent advances in source estimation and signal decomposition of multivariate surface potential and magnetic field measurements of the human brain. The aim of this paper is to identify current trends and to provide recent results related to the main research themes in this field.

Research attempts to answer questions such as: Where in the brain is an activity occurring? Are there multiple centers of activity for a given task? Is there a connection between the different activity centers? To answer these questions several procedural stages have to be accomplished: Is the measurement setup appropriate for the type of activity under investigation? Is the signal quality sufficient to draw meaningful conclusions? Does the spatial and temporal source model represent the known physiology? Is connectivity between activity centers plausible and observable? And finally, how can our conclusions be verified or validated?

Multichannel electro- or magnetoencephalographic (EEG, MEG) recordings taken non-invasively from outside the brain provide intricate multivariate data sets. To extract the information of interest, tools from rather diverse fields of mathematics are required such as time series analysis, modeling of the electromagnetic properties of the body tissues, and the estimation of sources in the brain. Both the temporal and spatial dimensions of these multivariate data sets appear equally important to retrieve knowledge about brain function.

For retrieving location, orientation, extent, shape, and strength of bioelectric sources in the brain, often only a single time instant of the measured EEG or MEG data is used, i.e., only the spatial dimension of the data is needed. This source reconstruction comprises the computation of bioelectric and biomagnetic fields in a sensor space owing to given 
configurations in a source space (forward problem) and the source parameter estimation based on given measurements (inverse problem) [35, 36]. In the low-frequency range (up to a few $\mathrm{kHz}$ ), which is the domain of bioelectricity, electric and magnetic fields can be described by the quasi-static Maxwell equations. This allows for a separate treatment of the generated electric and magnetic fields. Fortunately, only one important material parameter needs to be considered: the predominantly Ohmic tissue impedance. Another important simplification is the practically instantaneous propagation of electric and magnetic field. Accordingly, at a given instant in time and for a given set of bioelectric sources, one can assume that the sensor measurement constitutes a zero time delay and linear superposition of the source activity. In the past, the above listed considerations were combined into the spherical volume conduction model [35], which is superseded nowadays by far more advanced methods such as finite element (FE) modeling [27]. These models allow incorporation of well known physiological details such as anisotropic conductivity.

Information contained in the temporal dimension of the data can be used at least twofold. Firstly, the already mentioned superposition of sources can be resolved by the popular independent component analysis method (ICA) [18], which assumes statistical independence between the sources. This seems valid for a superposition of artifactual and brain sources. However, the view that several stationary brain sources embedded in passive tissue are always independent could be incomplete for several reasons. Thus, it appears obligatory to study to what extent the results of ICA are compromised if the condition of independence is not met. Secondly, connectivity between different brain areas [1,38] is probably the key to performance of the brain. Brain areas are connected through neural fibers, for which transmission speed is limited. Based on measurements in peripheral nerves, the upper limit of transmission velocity in nerve fibers is $120 \mathrm{~m} / \mathrm{s}$ [28]. This causes a time delay of at least $1 \mathrm{~ms}$ over a distance of $100 \mathrm{~mm}$ (e.g., across hemispheres). If electrical activity propagates through the mechanism of volume conduction, no time delay is visible. In other words, a non-zero time delay between two sources at different locations in the brain discovered by EEG or MEG is an important marker for functional connectivity. One approach of analyzing this marker is the multivariate autoregressive (MVAR) model and the derived coupling measures.

The extraction of parameters from multivariate data using advanced theoretical models in the time and spatial domain requires some form of validation. This is difficult for results related to brain function because of ethical reasons. For source reconstruction, phantom models are a valuable tool despite all their limitations, as it has been demonstrated for magnetocardiography (MCG) [43]. Connectivity results, however, deduced from measurements on the living brain are more difficult to validate directly. But secondary knowledge of anatomical or psychobehavioral nature can help to assess connectivity results.

As a broad range of topics is described in this paper the order of the sections is naturally not unique. To stress the importance of physiologically and technically sound experiments, the next section "Identification of deep sources in the brain" mainly describes the detection of weak brain signals, e.g., from deep subcortical sources, by EEG and MEG. The successful detection of several sources immediately leads to the question whether the sources are interrelated or coupled. This requires adequate coupling measures, which can be estimated using, e.g., MVAR, as presented in the section "The MVAR model with application to electroencephalogram and other biosignals". Any type of coupling violates the assumptions of the ICA method, probably limiting the scope of applicability of ICA. Therefore, synthetic MEG data with deliberately coupled sources are investigated in the section "Performance of ICA for dependent sources using synthetic stimulus evoked MEG data". Then in the section "EEG/MEG source analysis based on realistic finite element volume conductor models", FE modeling incorporating a precise description of anatomical and physical properties of the brain is used for source analysis. Finally, in the section "Assessment of source reconstruction methods", a case is made for the necessity to validate and verify source reconstruction methods using real measurements on phantoms and animals. Owing to space limitations and the topical organization of this research, numerous relevant general reviews could not be cited.

\section{Identification of deep sources in the brain}

Brain activity is a complex interplay between cortical areas and subcortical structures, such as thalamus, basal ganglia, hippocampus, and brain stem. Although the major contribution to EEG and MEG signals is usually generated by postsynaptic potentials of cortical pyramidal cells aligned in parallel [25], deeper structures below the cortex (e.g., thalamus, brainstem, basal ganglia) are also known to generate measurable signals [29]. However, these structures generally lack aligned pyramidal cells. Hence, it is unlikely that postsynaptic potentials contribute much to these signals. Trains of action potentials are therefore the most likely candidates for their generation [29]. The application of source localization algorithms to such signals is a challenge owing to low signal-to-noise ratios (SNRs) and rather unspecific scalp topographies. In the case of MEG, the increasing compensation of the primary currents by volume currents also contributes to the relative inaccessibility of deep sources. By contrast, an accurate localization of such sources would be very interesting, because it would help to better specify functional networks, e.g., by determining which thalamic nucleus is involved in a certain process. Moreover, because in some cases the locations of the potential sources are well known (e.g., ascending auditory pathway), such settings could be valuable for the validation of source localization techniques.

We took a closer look at source localization using the signals from MEG magnetometers and EEG electrodes. First, we performed a sensitivity analysis to find out how strong the activity has to be in order to be detectable outside the head. EEG/MEG sensitivity analyses have been performed 
previously. For example, lead field theory was used to compare the sensitivity profiles of different sensor configurations (pairs and triples of EEG electrodes, single MEG gradiometers and magnetometers) in a spherical volume conductor [26]. Here, we target the detectability of EEG and MEG signals with multi-sensor configurations and realistically shaped head models. As criterion for detectability, we assumed that the spontaneous background activity has a typical amplitude of $50 \mu \mathrm{V}$ for EEG and $300 \mathrm{fT}$ for MEG. A signal was considered detectable if in at least one channel the signal amplitude was twice the noise amplitude. Our analysis yields minimum source strengths (depending on precise source position and orientation) for EEG: cortex 50-150 nAm, hippocampus 175-650 nAm, thalamus and basal ganglia 175-550 nAm, and brain stem 200-650 nAm. For MEG, the sensitivities dramatically depend upon the dipole orientation. Although for the most favorable direction, we obtain minimum source strength of 10-15 nAm for the cortex and 25-150 nAm for most subcortical structures, unfavorable (i.e., radial) dipole directions can lead to 5-70 $\mu$ Am. Note that for averaged signals, the necessary dipole strength must be divided by the square root of the number of trials. A complex cognitive experiment is conducted with approximately 25-200 trials, whereas psychophysical measurements can feature 10,000 and more trials. It turns out that for EEG deep sources need to be approximately 4 times as strong compared to cortical sources. In normal cognitive experiments with up to 200 trials, this should lead to detectable signals also from subcortical sources. For MEG, in contrast, detectability is even better provided that the dipoles are maximally tangentially oriented, whereas both in the cortex [17] and in subcortical structures non-detectable sources are possible, particularly if dipole direction is rather radial, e.g., in the case of the ascending auditory pathway. Note that all numbers given above are subject to the assumed background noise levels and detectability criterion. Therefore, in reality the given minimum source strengths must be corrected by multiplication with the respective factors. There are several techniques to deal with initially bad SNRs. For example, prior information on the frequency spectrum or the coherence structure of the sources can be exploited by filtering techniques or frequency/coherence-specific reconstruction methods (e.g., DICS [14]). Moreover, subtracting suitable experimental conditions can improve the SNR of previously hidden signals [12]. If the overlaying unwanted signals (i.e., the noise) can be reasonably described as Gaussian and their covariance can be estimated with sufficient accuracy, Bayesian inference techniques [47] can be used to reconstruct the sources of the signal in spite of poor SNR. Another technique to improve the SNR, with particular emphasis to deep sources, has been proposed in Ref. [44].

Next, we recorded brain stem auditory evoked potentials and magnetic fields. To a healthy volunteer we delivered 30,000 chirp stimuli (short burst, changing frequency from low to high) to the right ear. As predicted by sensitivity analysis, the brain stem responses could be clearly observed in EEG, whereas it is more difficult to find responses in MEG [9]. This means that deep structures are observable with EEG, but only to a limited degree with MEG. In the section "EEG/MEG source analysis based on realistic finite element volume conductor models', it will be shown that it is possible to somewhat accurately localize the sources of brain stem auditory evoked potentials. The localization result shown in Figure 1 can only be achieved after a careful analysis of localization errors as a result of factors such as misspecification of skull and white matter anisotropy.

\section{The MVAR model with application to electroencephalogram and other biosignals}

An important key to the understanding of the human brain is the structure of connectivity in the brain. Although anatomical connections have been known for around 100 years,

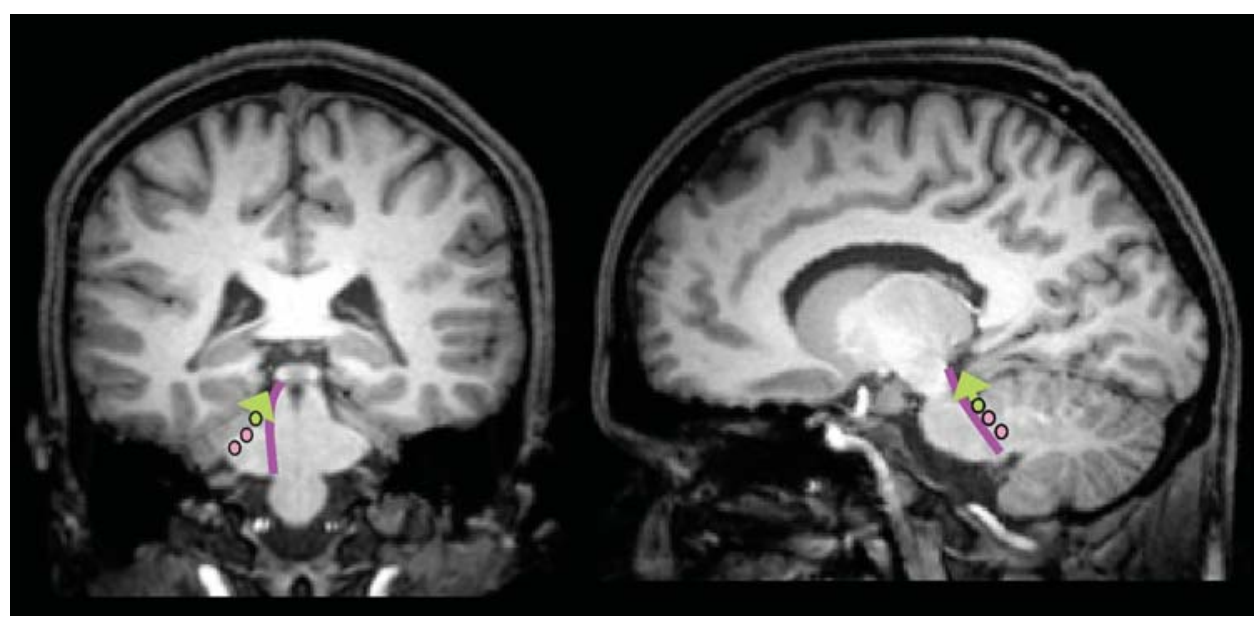

Figure 1 Localization of auditory brain stem potentials.

The dots represent localizations of wave V (7 ms after chirp offset) for different translation factors between diffusion anisotropy and conductivity anisotropy in the white matter (from low to high: 740, 1200, 1800); the green arrow indicates the dipole direction. The purple line denotes the course of the lateral lemniscus. 
the investigation of functional coupling between different brain areas is a much more recent topic. Methods probing coupling such as coherence (i.e., normalized cross-spectrum) have been in use on MEG and EEG data and related biosignals for more than 30 years. However, coherence has been criticized because the influence of volume conduction leads to spurious results. Several approaches, such as partial coherence, phase estimates, or imaginary coherence $[30,39]$ have been proposed as an alternative. Despite their advantages, these measures are unable to distinguish between direct and indirect connections (Figure 2). Nowadays, measures of Granger causality similar to the directed transfer functions and the partial directed coherence (PDC) can be used to address the issue of causal relationship. The first causality measure in the spectral domain was probably the directed transfer function (DTF [19]). However, in Ref. [1] it was shown that DTF cannot distinguish between direct and indirect connections; the PDC was proposed to solve this problem. If the time series are scaled differently, the PDC might yield incorrect causality; a refined version of PDC, the generalized PDC (GPDC [2]), addressed this problem.

The comparison of different measures is often complicated by the fact that different software tools are needed, each having their own implications and limitations. Fortunately, the MVAR model provides a common basis for analyzing the various coupling measures [39].

A MVAR model is described by Eq. (1), where $\vec{Y}_{t}$ are the observed time series of $m$ channels, $A_{m \times m}(k)=\left[a_{i, j}(k)\right]$ are $m \times m$ matrices of the model coefficients, $p$ is the order of the model, and $\vec{X}_{t}$ is the innovation process. It is a zero mean white noise process with covariance matrix $\Sigma_{X}=\left[\sigma_{i, j}\right]$. The parameters $a_{i, j}(k)$ model the influence of channel $j$ on channel $i$ with time delay $k$. Because the time delay is always larger than 0 , the model is a causal model, only past values influence the actual value:

$\vec{Y}_{t}=\sum A_{m \times m}(k) \cdot \vec{Y}_{t-k}+\vec{X}_{t}$

In the frequency domain, this corresponds to the transfer function $H(f)=\left[h_{i, j}\right]$ with:

$$
H_{m \times m}(f)^{-1}=A_{m \times m}(f)=I-\sum A_{m \times m}(k) \cdot \exp (-2 \pi \sqrt{-1} \cdot k \cdot f)
$$

The multivariate spectrum is defined as:

$$
S(f)=\left[S_{i, j}(f)\right]=H(f)^{-1} \cdot \Sigma_{X} \cdot H(f)
$$

and the coherency $C_{i, j}(f)$ between channels $i$ and $j$ is:

$$
C_{i, j}(f)=\frac{S_{i, j}(f)}{\sqrt{S_{i, i}(f) \cdot S_{j, j}(f)}}
$$

The standard PDC and the GPDC are defined as:

$$
\begin{aligned}
\operatorname{PDC}_{i, j}(f) & =\frac{A_{i, j}(f)}{\sqrt{\sum_{k}\left|A_{k, j}(f)\right|^{2}}} \\
\operatorname{GPDC}_{i, j}(f) & =\frac{\frac{1}{\sigma_{i, i}} A_{i, j}(f)}{\sqrt{\sum_{k} \frac{1}{\sigma_{k, k}^{2}}\left|A_{k, j}(f)\right|^{2}}}
\end{aligned}
$$

Several MVAR estimation algorithms are available and have been compared [38]. The MVAR-based analysis provides information on time-delayed correlations between sensors and the influence from channel $i$ to $j$ can be different from the influence of channel $j$ to $i$. This enables the distinction between forward and backward connections. If the model preconditions are preserved, the reconstruction of a causal structure can be achieved as demonstrated in Figure 2.

The imaginary coherence (iCOH) is antisymmetric, i.e., $\operatorname{imag}\left(C_{i, j}(f)\right)=-\operatorname{imag}\left(C_{j, i}(f)\right)$. If the $\mathrm{iCOH}$ is used as a coupling metric, a coupling from $i$ to $j$ has the same magnitude as coupling from $j$ to $i$. The PDC and GPDC are, in general, not symmetric and can describe a large coupling from $i$ to $j$ and a zero coupling in reverse direction. Figure 3 shows an example of a time-varying PDC during motor imagery task of a single subject. This PDC of real world data is not symmetric. The example shows a significant increase of PDC in the gamma range from $\mathrm{C} 3$ to $\mathrm{Cz}$ without a significant change from $\mathrm{Cz}$ to $\mathrm{C} 3$. These details cannot be identified by (anti)symmetric coupling measures such as $\mathrm{iCOH}$.

Unfortunately, volume conduction phenomena and the influence of a common reference electrode can cause an instantaneous mixture of activities. This causes an instantaneous correlation of the observed time series $\vec{U}_{t}=M \cdot \vec{Y}_{t}$ and a second MVAR model is obtained:

$\vec{U}_{t}=\sum B(i) \cdot \vec{U}_{t-i}+\vec{V}_{t}$

where the model parameters are $B(i)=M \cdot A(i) \cdot M^{-1}$ and the covariance of the innovation process becomes $\Sigma_{V}=M \cdot \Sigma_{X}$. $M^{T}$.

This mixing matrix influences all coupling parameters including PDC, iCOH, etc. and causes, typically, a "smearing effect". A significant non-zero iCOH indicates a functional coupling different from a spurious effect owing to volume conduction, but a proper localization is impossible without knowledge of the mixing matrix. The underlying network structure can only be identified if the mixing matrix $M$ is known and invertible. If some additional assumptions about the mixing matrix are introduced (e.g., a smearing effect is localized) these assumptions can be used to interpret the results from all metrics alike.

A possible mixing matrix satisfying Eq. (7) can be identified through eigenanalysis of $\Sigma_{V}$ or by ICA [11]. Unfortunately, there is not a unique solution and a manifold of 
A

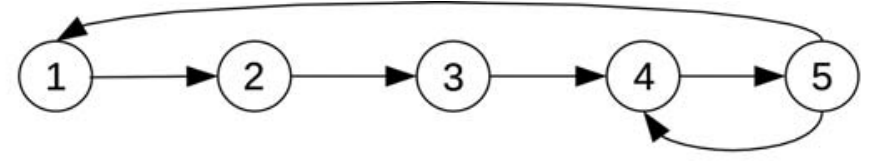

B

$\log ($ spectrum)

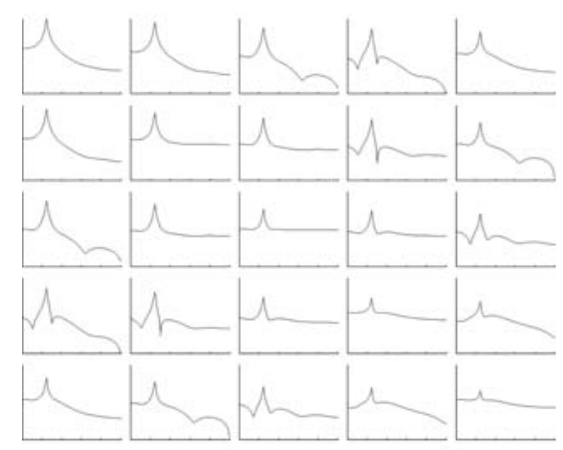

D

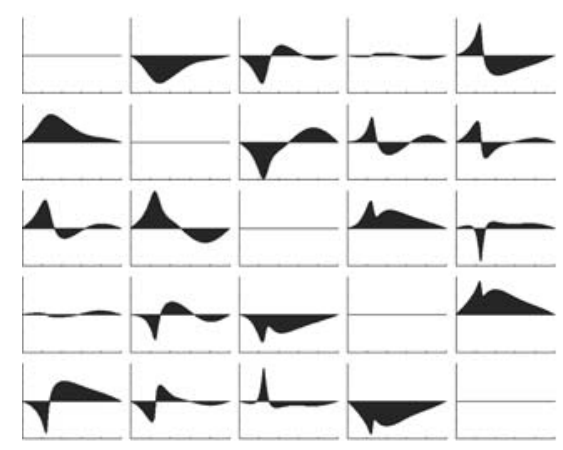

$\mathbf{F}$

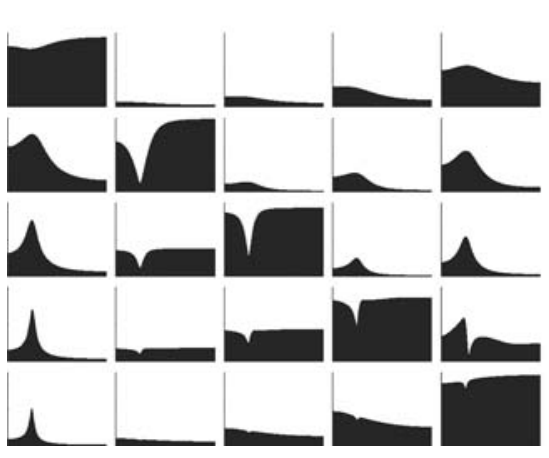

C

Coherence

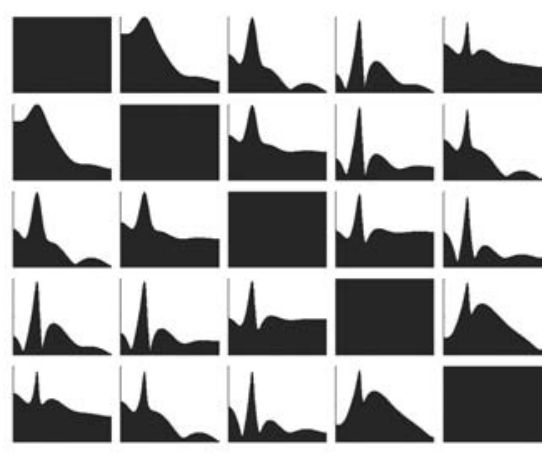

E Partial coherence

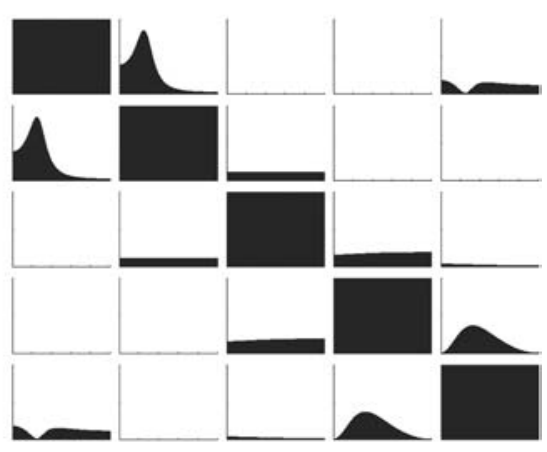

G

PDC

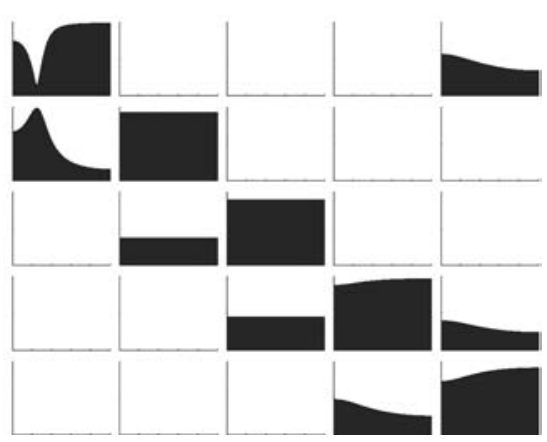

Figure 2 Coupling parameters from a 5-variate autoregressive model as defined in Ref. [2].

The MVAR model is based on the structure as defined in (A). (B) to (G) show the corresponding cross-spectra, coherence, imaginary coherence, partial coherence, directed transfer function (DTF), and partial directed coherence (PDC), respectively. Only PDC is able to reproduce the correct model structure in (A). Note that DTF and PDC are not symmetric and can distinguish between forward and backward connections. Biosig (http://biosig.sf.net) was used for this analysis (see demo7.m to reproduce the results of these and other coupling measures).

mixing matrices fulfill the above condition. Purely data-driven approaches cannot tell which unmixing matrix yields approximately correct source activations. Therefore, an estimate of the mixing matrix based on a priori information such as geometry and conductivity is required, i.e., a source local- ization procedure should be applied [42] to account for a possible volume conduction effect.

With advanced models such as those described in the section "EEG/MEG source analysis based on realistic finite element volume conductor models", the problem of volume 


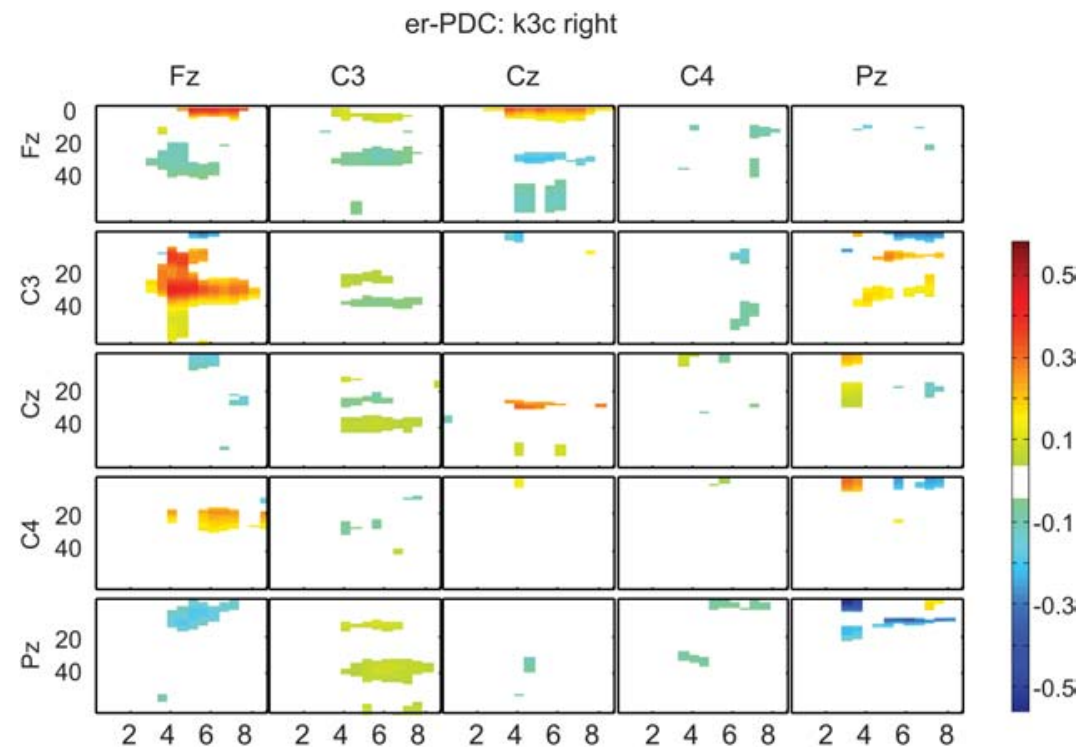

Figure 3 Example of a time frequency plot of the PDC for real data.

Electrode labels, time range (in $\mathrm{s}$ ), and frequency ranges (in $\mathrm{Hz}$ ) are indicated. The subject was instructed by a "cue" at $t=3 \mathrm{~s}$ to imagine a movement of the right hand. The PDC changes subsequently particularly in the $\gamma$ range $(30-50 \mathrm{~Hz})$ at electrode C3 (reproduced from Ref. [39], with permission from Elsevier, Amsterdam, The Netherlands).

conduction is alleviated particularly if coupling between cortical and subcortical sources is considered. In view of the shortcomings of MVAR-based methods, it should be noted that alternative methods are subject to the same limitations. Moreover, only the MVAR-based methods can distinguish direct from indirect causal relationships. This is a clear improvement for the characterization of functional brain connectivity.

\section{Performance of ICA for dependent sources using synthetic stimulus evoked MEG data}

Continuing with a topic related to connectivity, the consequence of coupled sources for models relying on statistical independence is discussed in this section. The class of algorithms known as ICA $[6,18,34,46]$ assumes statistical inde- pendence between sources to identify them in (typically) raw data without prior knowledge about either temporal or spatial properties of the sources. Considering the chirp experiment outlined in the section "Identification of deep sources in the brain", a sequence of brain areas will become activated by the auditory stimulus, i.e., different evoked sources can be triggered by the same stimulus. These sources will be coupled and the assumption of statistical independence could be violated. The performance of ICA for such a scenario of dependent evoked sources is currently unclear and synthetic data will be used to study this.

A synthetic MEG model [20] was introduced, namely the synthetic stimulus evoked jittered response (SSEJR) model, which allows generation of source signals with gradually adjustable dependencies. Synthetic MEG data are obtained by a processing chain as depicted in Figure 4. Two equivalent current dipoles (ECDs) describing evoked signal (black) and

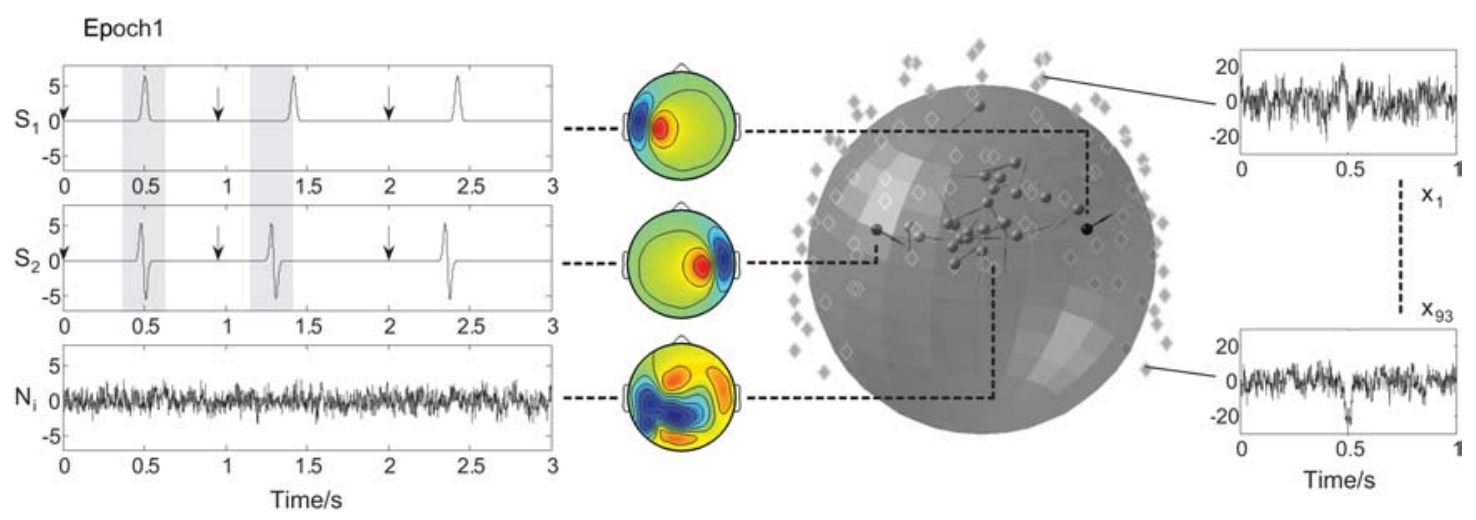

Figure 4 Generation of synthetic MEG data using the SSEJR model.

Two well-defined sources are combined with 23 noise sources. Using ECDs as forward model, synthetic MEG data are generated. 
23 ECDs describing noise (gray) are placed in a conducting sphere. Magnetic fields are calculated at MEG sensor coordinates (diamonds), using the quasi-stationary Maxwell equations [35]. The superposition of evoked and noise ECD field patterns yields synthetic MEG data, where each sensor records temporal dynamics corresponding to 100 generated epochs. Each epoch contains a response from the two sources to simulate neuronal activity following each individual stimulus. Choosing equal onset times (latencies) and equal durations of these responses leads to a correlation of the energy profiles of the source signals. Hence, the resulting source signals are dependent. Adding a zero mean normally distributed random number to the latencies of each source in each epoch leads to a jitter in latencies over epochs as illustrated in Figure 4 (left). The energy profiles are less correlated which lowers the dependency. The sequence of random numbers for each source latency has equal standard deviation, and the standard deviation will be referred to as jitter for convenience. The jitter gradually adjusts the degree of evoked source dependencies. The higher the jitter the lower the dependencies between the sources and vice versa. Figure 4 (middle) depicts the field maps obtained by forward calculation (the two shades of color define the polarity of the field), and Figure 4 (right) depicts typical generated sensor observations. These data should be used to assess the performance of ICA to identify the evoked MEG sensor space patterns, i.e., the field maps, at varying degrees of dependency.

Three established ICA methods (see abbreviations in Figure 5) as well as principal component analysis (PCA) have been tested to recover the sources, which here are meant to be sensor space maps and associated temporal functions and not ECDs as in the other sections. The Amari performance index is used to evaluate the ICA unmixing error. Specifically, the median unmixing error for 50 realizations of the SSEJR signals per jitter value is depicted in Figure 5. The median binned mutual information quantifies the source dependencies. It decreases gradually with increasing jitter, neatly confirming the SSEJR model. As expected, the per-

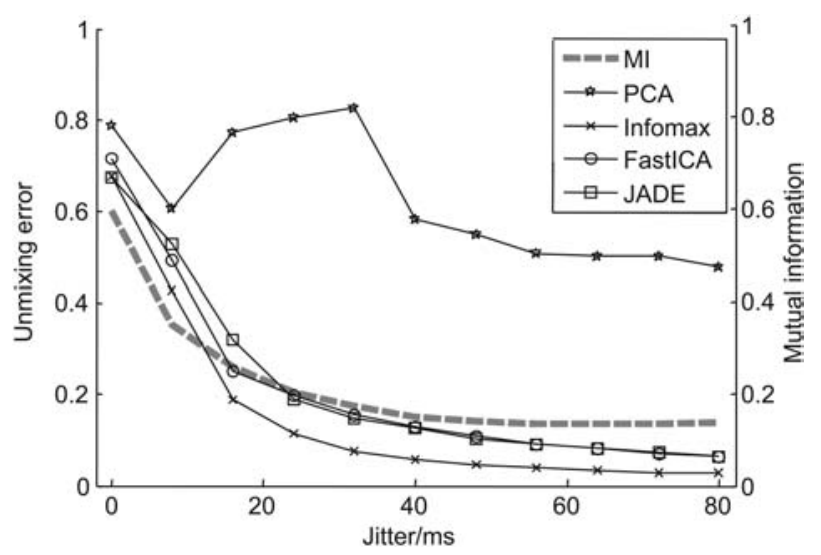

Figure 5 Performance of established ICA methods with regard to the jitter parameter in the SSEJR: for two dependent sources, i.e., at zero jitter, the unmixing error, and the mutual information are large reaching values of 0.75 .
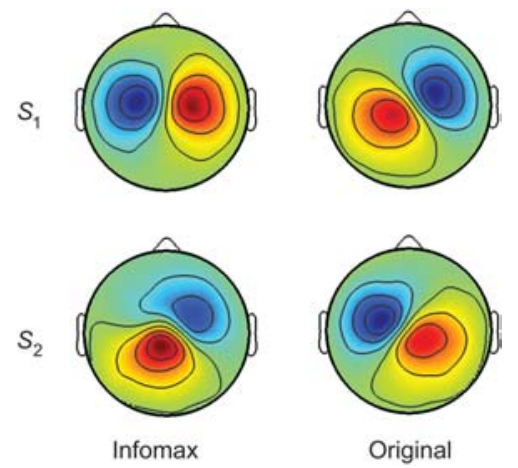

Figure 6 Example of closely spaced dipoles: the recovered field maps for dependent SSEJR sources do not correspond to the original sources.

formance of PCA is poor even in the vicinity of source independence. In contrast, the error for all ICA methods drops under 0.2 in this case and the ICA recovered sources equal almost the originals. However, it can be observed that all ICA methods lose performance when the jitter value is lowered. Strikingly, their unmixing error closely follows the estimated mutual information. Hence, ICA could fail to separate dependent evoked sources and the recovered signals as well as the associated field maps are still mixtures of the sources. Changing the sites of the source dipoles leads to another important aspect. For closely spaced dipoles, several ICA methods recovered clear dipolar patterns even for dependent sources. These results appear physiologically significant owing to their dipolar patterns but turned out to be incorrect, cf. Figure 6.

The numerical simulations with the SSEJR model show that ICA is sensitive to a violation of its key assumption, i.e., source independence. In the application of stimulus experiments, this could lead to ICA results that are still mixtures of the sources. Most critically, incorrect recovered ICA patterns can still show a dipolar structure. Hence, the common practice of judging an ICA decomposition to be successful when the estimated source patterns appear physiologically meaningful, i.e., show a dipolar pattern, could be misleading. A detailed source analysis such as that described in the next section might be helpful in classifying apparently dipolar ICA patterns.

\section{EEG/MEG source analysis based on realistic finite element volume conductor models}

This section describes an advanced source analysis with applications already suggested in the preceding sections. In Refs. $[10,16,21,23,24,27,33,40,43,49]$, it was shown to what extent spatial resolution of both EEG and MEG source analysis is dependent on the modeling accuracy of the embedded forward problem, i.e., the simulation of EEG and MEG fields for a given dipolar source in the brain using a volume conduction model of the head. Even if the irrotational source model proposed in Ref. [13] might be advantageous for the EEG forward problem because of a reduction 
of the number of unknowns, we use the standard concept of the current dipole model as proposed by Nolte et al. and Sarvas [30, 35], because we are particularly interested in developing methods for a combined analysis of EEG and MEG. Multimodal magnetic resonance imaging (MRI) and computed tomography registration and segmentation techniques are used for the generation of realistically shaped head volume conductor models. A low-resolution conductivity estimation method was developed [21] and a technique based on diffusion-tensor MRI was used [16, 31, 33, 49] to individually estimate head tissue conductivity inhomogeneity and anisotropy. Different FE techniques were developed for solving the forward problem $[3,8,22,27,37,45,48,50$, 51]. A key component in FE based source analysis is the numerical modeling of the singularity introduced into the differential equation by the current dipole and its interplay with the conductivity inhomogeneity and anisotropy.

The subtraction approach $[3,8,27,45,51]$ splits the total potential into two parts,

$\Phi=\Phi_{0}+\Phi_{\text {corr }}$

where the singularity potential, $\Phi_{0}$, is defined as the solution for a dipole in an unbounded homogeneous conductor with constant (anisotropic) conductivity $\sigma_{0}$ in a non-empty subdomain $\Omega_{0}$ around the source position (the so-called homogeneity condition; [8, 51]). The solution of Poisson's equation under these conditions for the singularity potential can be formed analytically for the mathematical dipole in an infinitely extended anisotropic medium [8, 51]. Subtracting this differential equation from the starting Poisson equation for the total potential yields a Poisson equation for the correction potential,

$-\nabla \cdot\left(\sigma \nabla \Phi_{c o r r}\right)=-\nabla \cdot\left(\left(\sigma_{0}-\sigma\right) \nabla \Phi_{0}\right)$,

with inhomogeneous Neumann boundary conditions at the head surface, $\left\langle\sigma \nabla \Phi_{\text {corr }}, n\right\rangle=-\left\langle\sigma \nabla \Phi_{0}, n\right\rangle$, and $\sigma$ being the conductivity and $n$ the outer normal vector at the head surface. The advantage of this reformulation is that the righthand side of the differential equation, Eq. (9), for the correction potential is free of any source singularity, because of the homogeneity condition: the conductivity $\sigma_{0}-\sigma$ is zero in $\Omega_{0}$. For the subtraction potential approach, a proof of existence and uniqueness was achieved for a weak solution in the function space of zero mean potential functions [51]. Furthermore, the convergence properties of the FE method have been proven for the numerical solution to the correction potential. A full subtraction approach was shown to achieve forward solutions, which were an order of magnitude more accurate compared to a projected subtraction approach [8]. An FE transfer matrix approach for both EEG and MEG was derived and its combination with algebraic multigrid preconditioned conjugate gradient solver techniques was shown to yield huge speedup factors and to enable mesh resolutions that seemed to be impossible previously $[22,50]$. Because of its ability to better model smooth tissue boundaries, an isoparametric FE approach in a geometry-adapted hexahedral model was shown to outperform an FE approach using regular hexahedral models [48]. Using modern inverse approaches, the developed methods were successfully applied in the fields of presurgical epilepsy diagnosis [33] and evoked responses [21].

FE-based dipole analysis was also used to localize the sources of the brain stem EEG signals of the section "Identification of deep sources in the brain'. For modeling the influence of the head tissue we used an FE model with five compartments for gray matter, white matter, cerebrospinal fluid, skull, and skin. The skull compartment was modeled anisotropically with different conductivities in radial and tangential directions [27]. The white matter was also modeled as anisotropic on the basis of diffusion tensor imaging [16, $33,49]$. We first investigated the impact of various model parameters onto the forward and inverse solutions associated to brain stem sources. For the scaling factor between diffusion anisotropy and conductivity anisotropy, we obtained a relative difference measure (RDM) up to 0.3 and localization errors up to $6 \mathrm{~mm}$. For skull anisotropy misspecifications, $\mathrm{RDM}$ up to 0.15 and localization errors up to $9 \mathrm{~mm}$ were observed.

The deviation of the volume currents in a realistic modeling approach from a simple spherical approach as used in the ECD model is immediately evident in Figure 7. It shows the volume currents for a thalamic dipole source computed in a finite element volume conductor model. Taking the data from the experiment described in the section "Identification of deep sources in the brain" it was possible to accurately localize a subcortical source (Figure 1). This demonstrates the potential of FE for reconstructing deep brain activity from EEG. The example also shows the impact of the white matter anisotropy, resulting in localization errors of several $\mathrm{mm}$, whereas orientation and magnitude errors might even be much more significant as shown previously $[15,16,33]$.

\section{Assessment of source reconstruction methods}

Inverse bioelectric and biomagnetic problems are computationally complex and do not have unique solutions. Consequently, assessment including validation and verification should be an inherent process not only for newly developed algorithms but also for new combinations of existing data preprocessing and data analysis procedures. In an engineering notation, verification means the internal quality control of the obtained result (e.g., the correct application of a data analysis chain under given boundary conditions or the crosscomparison between two different source localization methods), whereas validation means the quality assurance with regard to external evidence (e.g., comparing source localization results with known positions of invasive stimulation electrodes or stimulation electrodes in phantoms). Validation and verification approaches in general include simulations, phantom measurements, and in vitro and in vivo measurements. In the subclass of linear source reconstruction methods, this linearity can be exploited for assessment purposes with the help of resolution kernels [7, 12]. In vivo measure- 


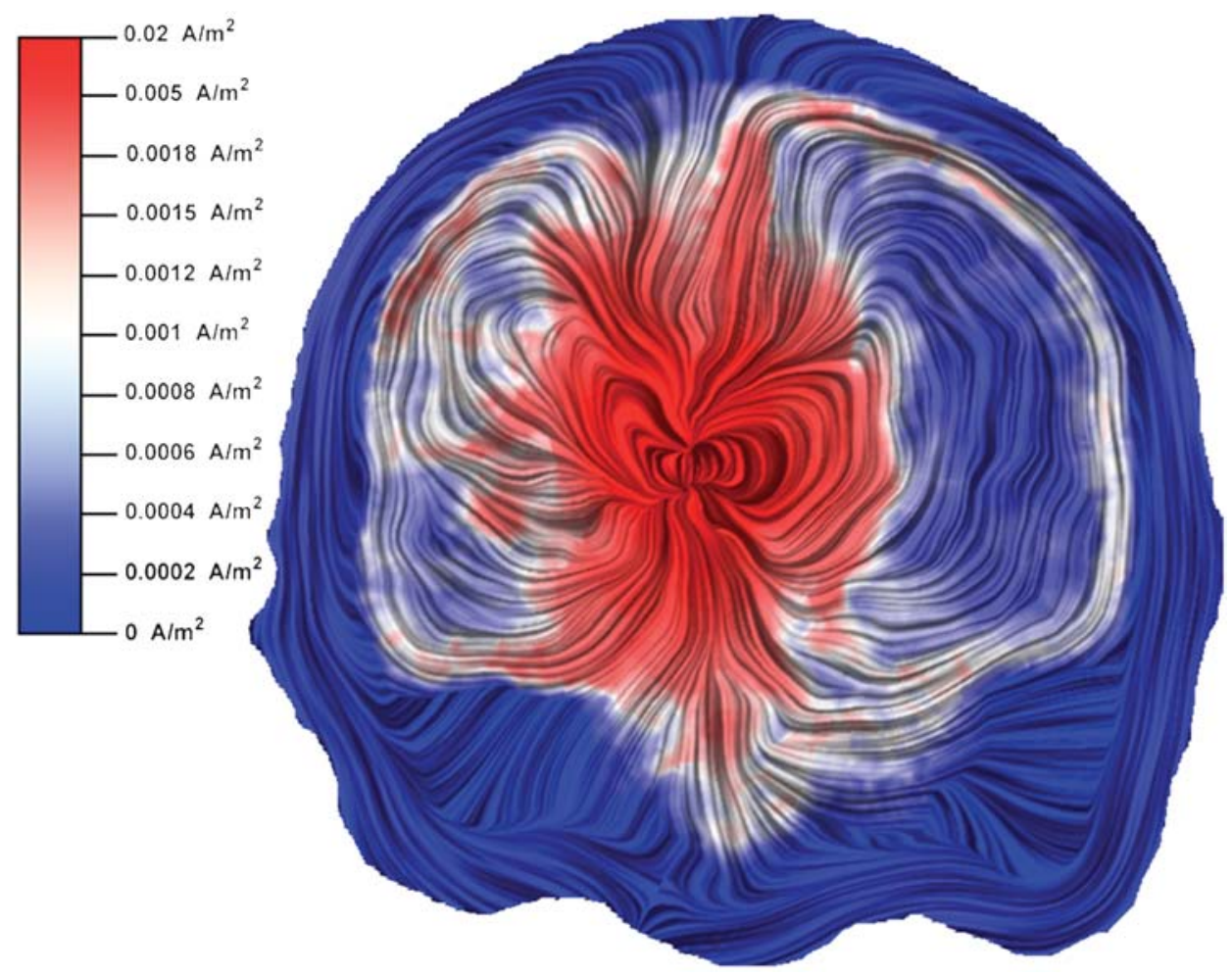

Figure 7 Volume currents for a thalamic dipole source computed in a finite element volume conductor model with 1:10 anisotropic white matter compartment, visualized on a coronal cut through the model.

(Reprinted from Ref. [49], with permission from Elsevier, Amsterdam, The Netherlands).

ments can be performed in animals [10] or humans [33]. A unique opportunity for in vivo validation or verification in humans is given by intracranial electrode setups in epileptic patients [33]. As an example the role of phantom and animal measurements for source localization will be discussed below.

Although simulations often provide a comparison with analytical models or between different numerical approaches $[22,37,41]$, only phantom, in vitro, and in vivo measurements use artificial dipoles and physiological stimulus modalities to determine procedural limits of the source localization accuracy under real world conditions. In the beginning of each validation or verification procedure, the definition of the problem and derivation of the requirements should be carried out. Based on that, a decision on the methodology, e.g., the simulation setup and the type of phantom, needs to be made. Often validation and verification are performed with a set of approaches. Such a line of validation or verification can, e.g., include simulations, phantoms of various degrees of complexity, and in vivo measurements. A typical example where such a line is recommended is the test of a newly developed inverse algorithm or a newly compiled data analysis chain.

As an example for the physiological stimulus modality the electric stimulation of peripheral nerves in sedated rabbits will be described. The median nerve was stimulated for the cortical representation of the forepaw and the tibial nerve for the cortical representation of the hindpaw (interstimulus interval of $500 \mathrm{~ms}$ and 2048 trials averaged). A 16-channel micro-superconducting quantum interference device (SQUID) MEG system was employed for recording the magnetic field. The electrocorticogram was recorded by a grid of $4 \times 4$ electrodes simultaneously over the contralateral hemisphere of the stimulated nerves. Data were sampled at a rate of $2 \mathrm{kHz}$ and preconditioned using a high pass filter of $0.3 \mathrm{~Hz}$ and a low pass filter of $300 \mathrm{~Hz}$. We focus our investigations on the first peak in the somatosensory evoked potential/field sequence, which is known as the first answer in the somatosensory cortex $\mathrm{S} 1$ and which is not overlaid by other cortical activities. Source localization was performed using both a boundary element model and a finite element model of the rabbit brain. A single current dipole fit was carried out for each of the stimulated cortical areas separately (used software: Curry V4.5, http://www.neuroscan.com/curry.cfm, and SIMBIO, https://www.mrt.uni-jena.de/simbio). The location of both cortical areas is known, and they have an inter-area distance of approximately $2 \mathrm{~mm}$. This is used to evaluate the results of the source localization procedures. We find that for peripheral nerve stimulation the localized sources in the rabbit brain are within an accuracy of $1 \mathrm{~mm}$ in the expected cortical areas. This holds for both the BEM and FEM models of the rabbit head and for both stimulated nerves. The difference between the cortical representations of the two nerves based on the source localization results is found to be $2.1 \mathrm{~mm}$. Similar accuracies are obtainable for source localization of peripheral nerve stimulation in swine 
[10]. In experiments with single artificial current dipoles in a saline filled rabbit skull phantom and in a saline filled spherical phantom of similar size we could show that the principal procedural source localization accuracy for our experimental setup is below $1 \mathrm{~mm}$.

We believe that physical phantom measurements provide a unique means to assess the performance of source localization techniques. Unlike simulations, they take into account the real world influences, such as environmental noise or 3D positioning errors, thus giving an error estimate of the entire source reconstruction procedure. Unlike in vivo measurements, no physiological uncertainties exist and the ground truth in terms of source position, strength, orientation, and extent is known. Limitations of phantom experiments are, e.g., the typically compartmental geometries owing to the constraints in mechanical construction, the restricted inclusion of biological noise sources, and the technical difficulties in constructing complex anisotropically conducting structures.

Phantom setups generally consist of a volume conductor, an artificial source, and a generator driving the source as will be described for MCG phantoms. To be compatible with magnetic measurement systems, non-magnetic materials such as resin with fiber glass or plastics were needed. We additionally embedded in our realistically shaped hollow torso phantom MCG compatible $\mathrm{Ag} / \mathrm{AgCl}$ electrodes for combined electric and magnetic measurements. The phantom was filled with saline solution with a fixed conductivity. An artificial dipole inside the torso driven by a sinusoidal current served as source. Separate compartments with differing conductivities, such as lungs, was used to assess the influence of these conductivity inhomogeneities onto the source localization results for focal dipolar sources [43]. Also the influence of anisotropic conductivity onto several aspects of magnetic fields and electric potentials was experimentally investigated. An improved realistic torso phantom equipped with artificial current dipole sources inside anisotropic compartments and in the vicinity of anisotropic compartments was developed $[24,40]$.

The estimation of the extent of active areas is of interest both in neuroscience and in cardiac applications. Here, phantom measurements can provide an objective measure of the ability of inverse algorithms to localize and describe extended sources [4]. Moreover, phantom experiments can be used to validate theoretical concepts, such as the vortex current concept (closed loop currents), which are considered as one reason for diverging information content in electric and magnetic signals $[5,32]$. With a torso phantom as described above and a set of dipoles forming a closed loop, the theoretically predicted differences between electric and magnetic signals could be quantified [23].

\section{Conclusion}

In this paper, we have presented recent results covering a broad range of EEG and MEG data analysis topics. In particular, the potential and the limits of some current state-of- the-art methods were discussed. For EEG and MEG, there exist clear sensitivity limits with regard to locating deep sources. This means in turn that less challenging problems can be readily solved. A similar reasoning holds for connectivity analysis methods and statistical signal processing using ICA: an optimal connectivity analysis with MVAR models depends on a reliable source reconstruction, but meaningful connectivity results can be obtained without source reconstruction. The independence assumption is very useful for artifact rejection using ICA, but with regard to interacting brain sources ambiguities occur in ICA results. Very detailed FE modeling is demonstrated and will help to reject or accept simpler forward solutions in case that only limited anatomical information is available. Validation is a key step in the assessment of an algorithm for a diagnostic application.

To date, most approaches for EEG and MEG data analysis are based exclusively on a temporal or spatial property of the signals, as are the methods discussed here. It is the hope that this compilation stimulates research into the combination of the advanced individual approaches for a unified view on the data. Examples could be the generation of time-dependent phantom data suitable for testing ICA or a connectivity analysis based on FE-modeled sources.

The necessity to understand various types of diseases of the brain on a functional level is as important as ever. Therefore, different tools as described here need to be combined with each other behind an easy-to-use interface suitable for a clinician. This requirement is fulfilled by other technologies applied for medical purposes. For EEG and MEG the software-based research methods developed by individuals and groups still have to be integrated in a coherent manner.

\section{Acknowledgements}

The authors gratefully acknowledge the following support. T.H.S.: Financial support by BMBF (Grant 01 GO 0208 Berlin Neuroimaging Center); T.R.K.: Moritz Dannhauer for contributing scripts and software and financial support by DFG (grants KN588/2 and KN588/3); F.K.: financial support by DFG (Grant OR 99/4-1); A.S.: Maren Grigutsch, MPI Leipzig, for pointing out possible problems of volume conduction for the PDC; C.H.W.: financial support by DFG (Grant WO1425/1-1 and JU445/5-1); J.H.: financial support by DFG Ha2899/6-1,8-1, BMBF 03IP605. The input given by two anonymous reviewers was of great help. There is no conflict of financial interest with third parties.

\section{References}

[1] Baccala LA, Sameshima K. Partial directed coherence: a new concept in neural structure determination. Biol Cybern 2001; 84: 463-474.

[2] Baccala LA, Takahashi DY, Sameshima K. Computer intensive testing for the influence between time series In: Schelter B, Winterhalder M, Timmer J, editors. Handbook of time series analysis - recent theoretical developments and applications. New York: Wiley 2006: 411-433.

[3] Bertrand O, Thevenet M, Perrin F. 3D finite element method in brain electrical activity studies. In: Nenonen J, Rajala HM, 
Katila T, editors. Biomagnetic localization and 3D modelling. Rep Dep Tech Phys, Helsinki University of Technology 1991: 154-171.

[4] Brauer H, Haueisen J, Ziolkowski M, Tenner U, Nowak H. Reconstruction of extended current sources in a human body phantom applying biomagnetic measuring techniques. IEEE Trans Magn 2000; 36: 1700-1705.

[5] Brockmeier K, Schmitz L, Bobadilla Cruz J, et al. Magnetocardiography and 32-lead potential mapping: the repolarization in normal subjects during pharmacologically induced stress. J Cardiovasc Electrophysiol 1997; 8: 615-626.

[6] Cichocki A, Amari S. Adaptive blind signal and image processing. New York: Wiley 2002.

[7] Di Rienzo L, Haueisen J, Arturi CM. Three component magnetic field data: impact on minimum morm solutions in a biomedical application. COMPEL 2005; 24: 869-881.

[8] Drechsler F, Wolters CH, Dierkes T, Si H, Grasedyck L. A full subtraction approach for finite element method based source analysis using constrained Delaunay tetrahedralisation. NeuroImage 2009; 46: 1055-1065.

[9] Erné SN, Scher HJ. Brainstem auditory evoked magnetic fields in the response to stimulation with brief tone pulses. Int J Neurosci 1987; 37: 115-125.

[10] Flemming L, Wang Y, Caprihan A, Eiselt M, Haueisen J, Okada Y. Evaluation of the distortion of EEG signals caused by a hole in the skull mimicking the fontanel in the skull of human neonates. Clin Neurophys 2005; 116: 1141-1152.

[11] Gómez-Herrero G, Atienza M, Egiazarian K, Cantero HL. Measuring directional coupling between EEG sources. NeuroImage 2008; 43: 497-508.

[12] Gonzalez Andino SL, Grave de Peralta Menendez R, Khateb A, Landis T, Pegna AJ. Electrophysiological correlates of affective blindsight. NeuroImage 2009; 44: 581-589.

[13] Grave de Peralta Menendez R, Gonzalez Andino SL, Morand $\mathrm{S}$, Michel CM, Landis T. Imaging the electrical activity of the brain: ELECTRA. Hum Brain Mapp 2000; 9: 1-12.

[14] Gross J, Kujala J, Hamalainen M, Timmermann L, Schnitzler A, Salmelin R. Dynamic imaging of coherent sources: studying neural interactions in the human brain. Proc Natl Acad Sci USA 2001; 98: 694-699.

[15] Güllmar D, Haueisen J, Eiselt M, et al. Influence of anisotropic conductivity on EEG source reconstruction: investigations in a rabbit model. IEEE Trans Biomed Eng 2006; 53: 1841-1850.

[16] Haueisen J, Tuch DS, Ramon C, et al. The influence of brain tissue anisotropy on human EEG and MEG. NeuroImage 2002; 15: $159-166$.

[17] Hillebrand A, Barnes GR. A quantitative assessment of the sensitivity of whole-head MEG to activity in the adult human cortex. NeuroImage 2002; 16: 638-650.

[18] Hyvärinen A, Karhunen J, Oja E. Independent component analysis. New York: Wiley 2001.

[19] Kaminski MJ, Blinowska KJ. A new method of the description of the information flow in the brain structures. Biol Cybern 1991; 65: 203-210.

[20] Kohl F, Wübbeler G, Kolossa D, Elster C, Bär M, Orglmeister R. Non-independent BSS: a model for evoked MEG signals with controllable dependencies. Proceedings of ICA 2009. Lect Notes Comp Sci 2009; 5441: 443-450.

[21] Lew S, Wolters CH, Anwander A, Makeig S, MacLeod RS. Improved EEG source analysis using low resolution conductivity estimation in a four-compartment finite element head model. Hum Brain Mapp 2009; 30: 2862-2878.

[22] Lew S, Wolters CH, Röer C, Dierkes T, MacLeod RS. Accuracy and run-time comparison for different potential approach- es and iterative solvers in finite element method based EEG source analysis. Appl Numer Math 2009; 59: 1970-1988.

[23] Liehr M, Haueisen J, Görnig M, Seidel P, Katila T, Nenonen J. Vortex shaped current sources in a Physical Torso Phantom. Ann Biomed Eng 2005; 33: 240-247.

[24] Liehr M, Haueisen J. Influence of anisotropic compartments on magnetic field and electric potential distributions generated by artificial current dipoles inside a torso phantom. Phys Med Biol 2008; 53: 245-254.

[25] Lopes da Silva F, van Rotterdam A. Biophysical aspects of EEG and magnetoencephalogram generation. In: Niedermeyer E, Lopes da Silva F, editors. Electroencephalography: basic principles, clinical applications, and related fields. 4th edition. Baltimore: Williams \& Wilkins 1999: 93-109.

[26] Malmivuo J, Suihko V, Eskola H. Sensitivity distributions of EEG and MEG measurements. IEEE Trans Biomed Eng 1997; 44: 196-208.

[27] Marin G, Guerin C, Baillet S, Garnero L, Meunier G. Influence of skull anisotropy for the forward and inverse problem in EEG: simulation studies using FEM on realistic head models. Human Brain Mapp 1998; 6: 250-269.

[28] Mills RG. Signaling in nervous systems. In: Bray JJ, Cragg PA, Macknight ADC, Mills RG, editors. Lecture notes on human physiology. 4th edition. Oxford: Blackwell Publishing 1999: 65-102.

[29] Murakami S, Okada Y. Contributions of principal neocortical neurons to magnetoencephalography and electroencephalography signals. J Phys Lond 2006; 575: 925-936.

[30] Nolte G, Bai O, Wheaton L, Mari Z, Vorbach S, Hallett M. Identifying true brain interaction from EEG data using the imaginary part of coherency. Clin Neurophysiol 2004; 115: 2292-2307.

[31] Olesch J, Ruthotto L, Kugel H, Fischer B, Wolters CH. A variational approach for the correction of field-inhomogeneities in EPI sequences. Proc SPIE (Medical Imaging 2010: Image Processing) 2010; 7623: in press.

[32] Roth BJ, Wikswo JP Jr. Electrically silent magnetic fields. Biophys J 1986; 50: 729-745.

[33] Rullmann M, Anwander A, Dannhauer M, Warfield SK, Duffy $\mathrm{FH}$, Wolters $\mathrm{CH}$. EEG source analysis of epileptiform activity using a $1 \mathrm{~mm}$ anisotropic hexahedra finite element head model. NeuroImage 2009; 44: 399-410.

[34] Sander TH, Burghoff M, Van Leeuwen P, Trahms L. Application of decorrelation independent component analysis to biomagnetic multi-channel measurements. Biomed Tech 2007; 52: 130-136.

[35] Sarvas J. Basic mathematical and electromagnetic concepts of the biomagnetic inverse problem. Phys Med Biol 1987; 32: $11-22$.

[36] Scherg M. Akustisch evozierte Potentiale: Grundlagen, Enstehungsmechanismen, Quellenmodell. Stuttgart: Kohlhammer 1992.

[37] Schimpf PH, Ramon CR, Haueisen J. Dipole models for the EEG and MEG. IEEE Trans Biomed Eng 2002; 49: 409-418.

[38] Schlögl A. A comparison of multivariate autoregressive estimators. Signal Processing 2006; 86: 2426-2429.

[39] Schlögl A, Supp G. Analyzing event-related EEG data with multivariate autoregressive parameters. Prog Brain Res 2006; 159: 135-147.

[40] Sengül G, Liehr M, Haueisen J, Baysal U. An experimental study on the effect of the anisotropic regions in a realistically shaped torso phantom. Ann Biomed Eng 2008; 36: 1836-1843.

[41] Stenroos M, Haueisen J. Boundary element computations in the forward and inverse problem of electrocardiography: com- 
parison of collocation and Galerkin weightings. IEEE Trans Biomed Eng 2008; 55: 2124-2133.

[42] Supp GG, Schlögl A, Trujillo-Barreto N, Müller MM, Gruber T. Directed cortical information flow during human object recognition: analyzing induced EEG gamma-band responses in brain's source space. PLoS ONE 2007; 2: e684.

[43] Tenner U, Haueisen J, Nowak H, Leder U, Brauer H. Source localization in an inhomogeneous physical thorax phantom. Phys Med Biol 1999; 44: 1969-1981.

[44] Väisänen O, Malmivuo J. Improving the SNR of EEG generated by deep sources with weighted multielectrode leads. J Physiol Paris 2009; 103: 306-314.

[45] van den Broek SP, Zhou H, Peters MJ. Computation of neuromagnetic fields using finite element method and Biot Savart Law. Med Biol Eng Comp 1996; 34: 21-26.

[46] Vigario R, Oja E. BSS and ICA in neuroinformatics: from current practices to challenges. IEEE Rev Biomed Eng 2008; 1 : $50-61$.

[47] Wipf DP, Owen JP, Attias HT, Sekihara K, Nagarajan SS. Robust Bayesian estimation of the location, orientation, and time course of multiple correlated neural sources using MEG. NeuroImage 2010; 49: 641-655.
[48] Wolters CH, Anwander A, Berti G, Hartmann U. Geometryadapted hexahedral meshes improve accuracy of finite element method based EEG source analysis. IEEE Trans Biomed Eng 2007; 54: 1446-1453.

[49] Wolters CH, Anwander A, Weinstein D, Koch M, Tricoche X, MacLeod R. Influence of tissue conductivity anisotropy on EEG/MEG field and return current computation in a realistic head model: a simulation and visualization study using highresolution finite element modeling. NeuroImage 2006; 30: 813-826.

[50] Wolters CH, Grasedyck L, Hackbusch W. Efficient computation of lead field bases and influence matrix for the FEM-based EEG and MEG inverse problem. Inverse Problems 2004; 20: 1099-1116.

[51] Wolters CH, Köstler H, Möller C, Härtlein J, Grasedyck L, Hackbusch W. Numerical mathematics of the subtraction method for the modeling of a current dipole in EEG source reconstruction using finite element head models. SIAM J Sci Comput 2007; 30: 24-45.

Received September 22, 2009; accepted February 26, 2010 